INPP-UVA-97-05

September 1997

\title{
New Relations and Constraints on Quark Spin-flavor Contents in Symmetry-breaking Chiral Quark Model
}

\author{
X. Song \\ Institute of Nuclear and Particle Physics \\ Department of Physics, University of Virginia \\ Charlottesville, VA 22901, USA \\ (September 7, 1997) \\ (Revised on November 13, 1997)
}

\begin{abstract}
New relations between the quark spin-flavor contents of the nucleon and axial weak coupling constants are obtained in the chiral quark model with both $\mathrm{SU}(3)$ and U(1)-breaking effects. Using the nonsinglet spin combinations, $\Delta_{3}$ and $\Delta_{8}$, all spin-flavor observables are functions of only one parameter $a-$ probability for the chiral pionic fluctuation. The upper and lower bounds of these observables are given. The optimum range of $a$, determined by NMC data $\bar{d}-\bar{u}$, gives a constraint to the cutoff of the chiral quark field theory. The model predictions are in good agreement with the existing data in this range of $a$. The roles of kaon, $\eta$ and $\eta^{\prime}$ are also discussed.
\end{abstract}




\section{Introduction}

In recent years, two interesting features of the spin-flavor structure of the nucleon have been revealed. First, the EMC [1] and later measurements [2 5] of polarized structure functions of the nucleon indicate that the quark spin fraction of the nucleon is unexpected small. It may be explained by a negative strange sea quark polarization, or the anomalous gluon contribution [6] in QCD modified quark parton model. Second, a strong violation of the Gottfried sum rule (GSR) [7], measured by the NMC group [8], implies that the downsea content exceeds over the up-sea $(\bar{d}-\bar{u}>0)$. The flavor asymmetry of the light quark sea distributions has also been confirmed in Drell-Yan process [9], which gives $\bar{u}(x) / \bar{d}(x) \simeq 0.5$ at $x=0.18$. Since the light quark masses are very small compared with the energy scale in deep inelastic processes, the $\bar{u} / \bar{d}$ asymmetry cannot be explained by the gluon splitting mechanism in the framework of the perturbative QCD. Since the gluon is flavorless, the quark sea generated by the gluon splitting is $\mathrm{SU}(3)$ symmetric in the LO QCD, while the NLO evolution only produces minor violation of SU(3) symmetry of sea. Considering the suppression arising from the strange quark mass effects, the SU(3) symmetry of the sea may be violated, but one expects that the $\mathrm{SU}(2)$ symmetry should hold due to the approximate equality $m_{u} \simeq m_{d}$. It is obvious that to understand these features, nonperturbative mechanism and the mass suppression effects are both needed. Historically, the Sullivan process [10] shown that the meson cloud of the nucleon can produce an excess of $\bar{d}$ over $\bar{u}$. This mechanism has been extensively used to explain the deficit of GSR. We only list a few earlier [11] and recent works [12 14]. Different models, for instance the Nambu-Jona-Lasinio (NJL) model, diquark model, and instanton model etc. were also suggested. A more detail list of references can be found in a review paper [15]. Among all efforts, the chiral quark model formulated in [16] seems provide a more promising framework to understand the nucleon structure. This model was first employed by Eichten, Hinchliffe and Quigg [17] to explain both the sea flavor asymmetry and the smallness of the quark spin fraction. The SU(2) chiral quark model calculation leads to an one-parameter fit to the sea flavor asymmetry and the quark spin contents. However, a full U(3) symmetry calculation produces a flavor symmetric sea. To solve this problem, the U(1)-breaking was introduced by Cheng and Li in 18 . They obtained a rather good two-parameter fit to the spin-flavor contents of the nucleon. However, the $0^{-}$Goldstone boson emission with $\mathrm{SU}(3)$ symmetry predicts $f_{3} / f_{8}=1 / 3$ and $\Delta_{3} / \Delta_{8}=5 / 3$ (the definitions of $\Delta_{3,8}$ and $f_{3,8}$ can be found in (2.1) and (2.8e) in Section II below), which are inconsistent with the experimental data. To remove this inconsistency, the $\mathrm{SU}(3)$ breaking effect arising from the suppression of chiral kaonic fluctuation was first introduced in [19]. A more generalized version which includes the $\eta$ and $\eta^{\prime}$ suppression effects was subsequently given in [20]. A somewhat different mass suppression description with $\lambda_{8^{-}}$ breaking was studied in 21]. The results shown that the kaonic suppression is important not only for removing the inconsistency in $\mathrm{SU}(3)$ symmetry description, but also for obtaining a better fit to the data. We note that a generalized version including suppression effects arise from $\eta$ and $\eta^{\prime}$ fluctuations was also obtained in [22]. The previous works need to be improved due to (1) the NA51 data $\bar{u}(x) / \bar{d}(x)$ at $x=0.18$ was assumed to be equal to the ratio of $\bar{u} / \bar{d}$, and this is highly questionable, (2) there is no definite rule to determine the parameters, and it was not clear which data we should consider first in verifying the model predictions. In addition, the previous comparison of the model prediction with data, especially in the quark flavor sector, also needs to be improved. These issues will be addressed in this paper. The 
main results given in the work [20] are briefly reviewed in section I. Using the nonsinglet spin combinations $\Delta_{3}$ and $\Delta_{8}$, the description given in 20] is reformed in section II, and all spin-flavor observables depend only on one parameter. In this description, new relations and constraints on quark spin and flavor contents are obtained. Numerical results, discussions and brief summary are given in sections III.

\section{The chiral quark model}

The effective chiral quark model describes the nucleon properties in the scale range between $\Lambda_{\chi \mathrm{SB}}(\sim 1 \mathrm{GeV})$ and $\Lambda_{\mathrm{QCD}}(\sim 0.2-0.3 \mathrm{GeV})$, where the spontaneously breaking of chiral symmetry leads to the existence of Goldstone bosons (GB). The important degrees of freedom are the constituent (dressed) quarks and Goldstone bosons, and the dominant interaction is the coupling among the quarks and Goldstone bosons, while the gluon effect is expected to be rather small. In the chiral fluctuation process, a quark could change its spin and flavor by emitting Goldstone bosons. Hence the spin-flavor contents of the nucleon are determined by the valence quark structure and all possible chiral fluctuations. The light quark sea asymmetry $\bar{u}<\bar{d}$ is attributed to asymmetric $\pi^{+}$and $\pi^{-}$fluctuations, which are originated from the existing flavor asymmetry of the valence quark numbers in the proton. The quark spin reduction, on the other hand, is due to the spin dilution in the chiral fluctuation processes $q_{\uparrow} \rightarrow q_{\downarrow}+G B$.

\section{SU(3)-breaking from kaonic suppression}

The SU(3)-breaking effect arising from the kaonic suppression was studied in [19]. The probability of the chiral fluctuation $u \rightarrow d \pi^{+}$or $d \rightarrow u \pi^{-}$is defined by

$$
a \equiv\left|g_{8}\right|^{2}=\left|\Psi\left(u \rightarrow \pi^{+} d\right)\right|^{2}=\left|\Psi\left(d \rightarrow \pi^{-} u\right)\right|^{2}
$$

where $g_{8}$ is the quark-octet meson coupling in the $\mathrm{SU}(3)$ symmetry description. The $\mathrm{U}(1)$ breaking parameter is $\zeta=g_{0} / g_{8} \neq 1$, where $g_{0}$ denotes the quark- $\eta^{\prime}$ coupling. The $\mathrm{SU}(3)$ breaking parameter $\epsilon$ is defined by [19]

$$
\epsilon=\left|\Psi\left(u \rightarrow K^{+} s\right)\right|^{2} /\left|\Psi\left(u \rightarrow \pi^{+} d\right)\right|^{2}
$$

which is the ratio between the probabilities of the chiral kaonic and pionic fluctuations. Since the chiral kaon is presumably more massive than chiral pions, the amplitude for emitting a kaon from a light quark is suppressed and thus $\epsilon<1$. Considering the first order chiral fluctuation, the results were [19]

$$
\begin{gathered}
\bar{u}=\frac{a}{3}\left(\zeta^{2}+2 \zeta+6\right) \\
\bar{d}=\frac{a}{3}\left(\zeta^{2}+8\right) \\
\bar{s}=\frac{a}{3}\left(\zeta^{2}-2 \zeta+10\right)-3 a(1-\epsilon)
\end{gathered}
$$

and

$$
\Delta u=\frac{4}{3}-\frac{1}{9}\left(8 \zeta^{2}+37\right) a+\frac{4 a}{3}(1-\epsilon)
$$




$$
\begin{gathered}
\Delta d=-\frac{1}{3}+\frac{2}{9}\left(\zeta^{2}-1\right) a-\frac{a}{3}(1-\epsilon) \\
\Delta s=-a+a(1-\epsilon)
\end{gathered}
$$

For a reasonable value $\epsilon \simeq 0.5$ and the parameters $(a \simeq 0.10, \zeta \simeq-1.2)$ used in $18 \|$, we obtained $f_{3} / f_{8} \simeq 0.26$ and $\Delta_{3} / \Delta_{8} \simeq 1.94$, which are much closer to the data. As mentioned in [19], the $\mathrm{SU}(3)$ breaking effect arising from the kaonic suppression is the key factor to break the $\mathrm{SU}(3)$ results $f_{3} / f_{8}=1 / 3$ and $\Delta_{3} / \Delta_{8}=5 / 3$, and change them in the right direction. Taking $\epsilon \rightarrow 1$, all $(1-\epsilon)$ terms vanish, thus (1.2a-c) and (1.3a-c) reduce into the $\mathrm{SU}(3)$ symmetry results given in [18].

$S U(3)$-breaking including $\eta$ and $\eta^{\prime}$ suppression effects

Having studied the breaking effect of chiral kaonic suppression, generalizing to include the $\eta$ and $\eta^{\prime}$ suppression is straightforward and the results were given in 20

$$
\begin{gathered}
u=2+\bar{u}, \quad \bar{u}=\frac{a}{9}\left[9+2(3-A)^{2}+A^{2}\right] \\
d=1+\bar{d}, \quad \bar{d}=\frac{a}{9}\left[18+2 A^{2}+(3-A)^{2}\right] \\
s=0+\bar{s}, \quad \bar{s}=\frac{a}{3}\left[9+B^{2}-9(1-\epsilon)\right]
\end{gathered}
$$

and

$$
\begin{gathered}
\frac{\bar{u}}{\bar{d}}=1-\frac{2 A}{(A-1)^{2}+8} \\
\bar{d}-\bar{u}=a \frac{2 A}{3}
\end{gathered}
$$

where

$$
A \equiv 1-\zeta^{\prime}+\frac{1-\sqrt{\epsilon}_{\eta}}{2}, \quad B \equiv \zeta^{\prime}-\sqrt{\epsilon}_{\eta}
$$

The parameters $\epsilon_{\eta}$ and $\epsilon_{\eta^{\prime}}$ were defined as

$$
\left|\Psi\left(u \rightarrow \eta\left(\eta^{\prime}\right) u\right)\right|^{2}=\left|\Psi\left(d \rightarrow \eta\left(\eta^{\prime}\right) d\right)\right|^{2} \equiv \epsilon_{\eta\left(\eta^{\prime}\right)} a
$$

where $\zeta^{\prime}=\sqrt{\epsilon_{\eta^{\prime}}} \zeta$. Physically, $\zeta^{\prime}$ combines both U(1)-breaking (other than mass effect) and mass suppression effect in the $\eta^{\prime}$ fluctuation. Hence $\zeta^{\prime}$ can be treated as an effective $\mathrm{U}(1)$-breaking parameter.

The results for the spin contents were 20

$$
\begin{gathered}
\Delta u=\frac{4}{3}-\frac{a}{9}\left(8 \zeta^{\prime 2}+37\right)+\frac{4 a}{3}(1-\epsilon)+\frac{4 a}{9}\left(1-\epsilon_{\eta}\right) \\
\Delta d=-\frac{1}{3}+\frac{2 a}{9}\left(\zeta^{\prime 2}-1\right)-\frac{a}{3}(1-\epsilon)-\frac{a}{9}\left(1-\epsilon_{\eta}\right) \\
\Delta s=-a+a(1-\epsilon)
\end{gathered}
$$

Taking $\epsilon_{\eta, \eta^{\prime}} \rightarrow 1$ and $\zeta^{\prime} \rightarrow \zeta,(1.2 \mathrm{a}-\mathrm{c})$, and $(1.3 \mathrm{a}-\mathrm{c})$ can be easily recovered, where the $\mathrm{SU}(3)$ breaking effect arises from the kaon suppression only. Assuming $\epsilon_{\eta} \simeq \epsilon\left(m_{\eta} \simeq m_{K}\right)$ 
and using data $\bar{d}-\bar{u}$ and a $\bar{u} / \bar{d}$ value estimated from some phenomenological antiquark distributions, a good agreement between the model predictions and data was obtained [20].

Several remarks should be made here. (1) The strange quark polarization $\Delta s$ is not affected by introducing the suppression of $\eta$ and $\eta^{\prime}$ mesons, because the $\eta$ and $\eta^{\prime}$ are all strangeness-0 and spin-0 mesons. They provide equal components of $s_{\uparrow}$ and $s_{\downarrow}$ and give vanishing contribution to the strange spin content. Only nonvanishing contribution to $\Delta s$ is coming from the kaons, and it decreases the strange flavor content $\bar{s}$ by $3 a(1-\epsilon)$, and reduce the magnitude of the strange quark polarization $\Delta s$ by $a(1-\epsilon)$ as given in [19]. However, the suppression effects of chiral $\eta$ and $\eta^{\prime}$ fluctuations do give some contributions to nonstrange quark polarizations: $\Delta u$ becomes more positive and $\Delta d$ is more negative. This certainly gives a better fit to the data. (2) The special combinations $A$ and $B$, appeared in quark flavor contents (1.4a-c), do not appear in (1.8a-c). This is because the $\eta$ and $\eta^{\prime}$ are spin-0 neutral mesons, the $A, B$ terms appeared in the spin-up quark content $\left(q_{\uparrow}\right)$ are completely the same as those in the spin-down case $\left(q_{\downarrow}\right)$, hence they cancel each other in $\Delta q$. (3) All antiquarks in the sea are unpolarized

$$
\Delta \bar{u}=0, \quad \Delta \bar{d}=0, \quad \Delta \bar{s}=0
$$

which hold for all chiral quark models with only first order Goldstone boson fluctuations, because the Goldstone bosons consist of equal components of $\bar{q}_{\uparrow}$ and $\bar{q}_{\downarrow}$ This prediction is consistent with recent semi-inclusive data 23] (see Table III), and has been used to explain the baryon magnetic moments [24].

Since (1.8c) provides a simple relation between $\Delta s$ and the parameters $a$ and $\epsilon$, it is tempting to use (1.8c) and the data $\Delta s$ to determine $\epsilon$, if one has obtained $A$ and $a$ from (1.5a-b) by using data $\bar{u} / \bar{d}$ and $\bar{d}-\bar{u}$. However, the data given by NA51 experiment [9] only provides one value of the ratio $\bar{u}(x) / \bar{d}(x)$ at $x \simeq 0.18$, while we need a ratio of the overall integrated quantities $\bar{u}$ and $\bar{d}$, which is almost undetermined. In addition, $\Delta s$ measured in the deep inelastic polarized lepton nucleon scattering is less precisely known. In Section II, a new approach and an one-parameter description will be introduced.

\section{New relations and constraints}

Let us reform the formalism $(1.8 \mathrm{a}-\mathrm{c})$ and $(1.4 \mathrm{a}-\mathrm{c})$, and rewrite the observables of spinflavor contents as functions of one parameter $a$ only. Defining the nonsinglet spin combinations, or nonsinglet axial charges

$$
\Delta_{3} \equiv \Delta u-\Delta d, \quad \Delta_{8} \equiv \Delta u+\Delta d-2 \Delta s
$$

and assuming $\epsilon_{\eta}=\epsilon$, from $(1.8 \mathrm{a}-\mathrm{c})$, one obtains

$$
\begin{gathered}
\zeta^{\prime 2}=\frac{1}{2 a}\left[3\left(1-\Delta_{8}\right)-\Delta\right]-\frac{7}{2} \\
\epsilon=1-\frac{\Delta}{2 a}
\end{gathered}
$$

where

$$
\Delta \equiv \frac{3}{5} \Delta_{3}-\Delta_{8}
$$


In the $\mathrm{SU}(6)$ valence quark model, $\Delta u=4 / 3, \Delta d=-1 / 3, \Delta s=0$, and $\Delta_{3}=5 / 3, \Delta_{8}=1$, which lead to $\Delta=0$. Hence $\Delta \neq 0$ measures the deviation from the naive quark model prediction. According to Cabibbo's description, the nonsinglet axial charges $\Delta_{3}$ and $\Delta_{8}$, related to the weak axial couplings, can be measured in the hyperon $\beta$-decays [25] 28],

$$
\begin{gathered}
\Delta_{3}=\Delta u-\Delta d=\left(\frac{G_{A}}{G_{V}}\right)_{n \rightarrow p}=F+D \\
\Delta_{8}=\Delta u+\Delta d-2 \Delta s=3 F-D
\end{gathered}
$$

Experimentally, they are rather precisely known

$$
\Delta_{3}=1.2573 \pm 0.0028, \quad \Delta_{8}=0.579 \pm 0.025
$$

hence

$$
\Delta=0.175 \pm 0.025
$$

which is about $15-20 \%$ deviation from zero. Having known $\Delta_{3}, \Delta_{8}$ and $\Delta$, from (2.2a-b) and (1.6), all spin and flavor contents are now functions of one parameter a only.

New relations on quark spin contents

Using (2.2a-b), the quark spin contents $(1.8 \mathrm{a}-\mathrm{c})$ can be rewritten as

$$
\begin{gathered}
\Delta u=\frac{4}{5} \Delta_{3}-a \\
\Delta d=-\frac{1}{5} \Delta_{3}-a \\
\Delta s=\frac{\Delta}{2}-a
\end{gathered}
$$

and the total quark spin is

$$
\Delta \Sigma=\Delta u+\Delta d+\Delta s=\frac{3}{5} \Delta_{3}+\frac{\Delta}{2}-3 a
$$

Equations (2.7a-d), derived from the chiral quark model with both $\mathrm{SU}(3)$ and axial U(1) breakings, provide new and simple relations connecting the quark spin contents and weak axial couplings. The comparison with the Skyrme model [29] and naive quark model results is shown in Table 1.

Observables related to the quark flavor contents

(a) The ratio of total antiquark contents to total quark contents is

$$
r_{\bar{q} / q} \equiv \frac{\sum \bar{q}}{\sum q}=\frac{1}{2}\left(1-\frac{1}{K_{1}+1+3 a}\right), \quad K_{1} \equiv 1-\Delta_{8}-3 \Delta / 2
$$

(b) The ratio of the total strange sea to the light antiquark contents is

$$
r_{2 \bar{s} / \bar{u}+\bar{d}} \equiv \frac{2 \bar{s}}{\bar{u}+\bar{d}}=\frac{B^{2}+9(1-\Delta /(2 a))}{A^{2}-3 A+9}
$$


(c) The ratio of the total strange sea to the light quark contents is

$$
r_{2 \bar{s} / u+d} \equiv \frac{2 \bar{s}}{u+d}=2 a \frac{B^{2}+9(1-\Delta /(2 a))}{9+2 a\left(A^{2}-3 A+9\right)}
$$

(d) The ratio of the total strange sea to total quark and antiquark contents is

$$
f_{s} \equiv \frac{s+\bar{s}}{\sum(q+\bar{q})}=\frac{2 a}{9} \frac{B^{2}+9(1-\Delta /(2 a))}{K_{1}+1+3 a}
$$

(e) Defining $f_{q} \equiv(q+\bar{q}) / \sum(q+\bar{q}), f_{3} \equiv f_{u}-f_{d}$ and $f_{8} \equiv f_{u}+f_{d}-2 f_{s}$, one has

$$
\frac{f_{3}}{f_{8}} \equiv \frac{u-d+\bar{u}-\bar{d}}{u+d-2 s+\bar{u}+\bar{d}-2 \bar{s}}=\frac{1}{3}\left(1+K_{2}\right)^{-1}
$$

with $K_{2} \equiv 4 a\left[\left(A^{2}-B^{2}\right)+9 \Delta /(2 a)\right] /(1-4 a A / 3)$, where $A$, and $B$ are functions of $a$ determined from (1.6), and (2.2a-b). Taking $\epsilon, \epsilon_{\eta} \rightarrow 1$, then $\Delta \rightarrow 0, A \rightarrow-B$, one has $K_{2} \rightarrow 0$ and $f_{3} / f_{8} \rightarrow 1 / 3$.

(f) There is a relation among the observables given in $(2.8 \mathrm{~b}-\mathrm{d})$

$$
f_{s}=\left(1+\frac{1}{r_{2 \bar{s} / u+d}}+\frac{1}{r_{2 \bar{s} / \bar{u}+\bar{d}}}\right)^{-1} \text {. }
$$

To obtain the upper and lower bounds of the spin and flavor observables, we need to discuss the physically allowed range of $a$ and the sign of $\zeta^{\prime}$.

\section{Parameter a}

Since $\zeta^{\prime 2}$ and $\epsilon$ must be positive, from $(2.2 \mathrm{a}-\mathrm{b})$ one obtains the lower and upper bounds of $a$

$$
a_{\min }=\frac{\Delta}{2}, \quad a_{\max }=\frac{1}{7}\left[3\left(1-\Delta_{8}\right)-\Delta\right]
$$

Using (2.5) and (2.6), we have $a_{\min }=0.088 \pm 0.012$ and $a_{\max }=0.155 \pm 0.004$, hence the allowed range of $a$ is approximately

$$
0.08<a<0.16
$$

In section III, we will show that this range can be further narrowed by using NMC data $\bar{d}-\bar{u}$. One interesting prediction, from $(2.7 \mathrm{c})$, is that the upper bound of the strength of the strange quark polarization is

$$
|\Delta s|_{\max }=a_{\max }-\frac{\Delta}{2} \simeq 0.08
$$

The probability $a$ for an up-quark splitting into a down-quark and a $\pi^{+}$can be estimated in the chiral field theory [17]

$$
a=\frac{g_{A}^{2} m^{2}}{8 \pi^{2} f_{\pi}^{2}} \int_{0}^{1} z d z \Theta\left(z_{\max }-z\right)\left\{\ln \left[\frac{\Lambda^{2}+m_{\pi}^{2}}{\tau(z)+m_{\pi}^{2}}\right]+m_{\pi}^{2}\left[\frac{1}{\Lambda^{2}+m_{\pi}^{2}}-\frac{1}{\tau(z)+m_{\pi}^{2}}\right]\right\}
$$


where $g_{A} \simeq 0.75$ is dimensionless axial-vector coupling, $f_{\pi} \simeq 0.093 \mathrm{GeV}$ the pion decay constant, $m \simeq 0.35 \mathrm{GeV}$ the constituent mass of $u$ or $d$-quarks, $m_{\pi}$ the pion mass, $\Lambda$ the ultraviolet cutoff, $\tau(z)=m_{u}^{2} z^{2} /(1-z)$ and $z_{\max }=\frac{\Lambda^{2}}{2 m^{2}}\left(\sqrt{1+\frac{4 m^{2}}{\Lambda^{2}}}-1\right)$. The range given in (2.9b) can be well reproduced by taking $\Lambda \simeq 1.20-2.57 \mathrm{GeV}$.

Sign of $\zeta^{\prime}$

From $(1.5 \mathrm{~b}),(1.6)$, and $(2.2 \mathrm{a}-\mathrm{b})$, one obtains $\zeta^{\prime}$ as function of $a$

$$
\zeta^{\prime}=1-\frac{3 \delta}{2 a}+\frac{1}{2}\left[1-\sqrt{1-\frac{\Delta}{2 a}}\right]
$$

where $\delta \equiv \bar{d}-\bar{u}$ is input. we plot the $\zeta^{\prime}-a$ curves for $\delta=0.130,0.147$, and 0.164 , in Fig. 1. Here and in what follows we literally use the central value of NMC result $\bar{d}-\bar{u}=0.147$ and restrict the error to \pm 0.017 in the numerical evaluation (see discussion of possible large error on (3.1) in section III). For the allowed range of $a$ given in (2.9b), the U(1)-breaking parameter $\zeta^{\prime}$ is negative.

Range of $\epsilon$

From $(2.2 \mathrm{~b})$, one has

$$
1-\frac{\Delta}{2 a_{\min }}<\epsilon<1-\frac{\Delta}{2 a_{\max }}
$$

or

$$
0<\epsilon<1-\frac{a_{\min }}{a_{\max }}
$$

Hence the upper bound of $\epsilon$ is approximately 0.43 .

Ranges of $\Delta u,|\Delta d|,|\Delta s|$ and $\Delta \Sigma$

From $(2.7 \mathrm{a}-\mathrm{d})$, we obtain

$$
\begin{aligned}
& \frac{4}{5} \Delta_{3}-a_{\max }<\Delta u<\frac{4}{5} \Delta_{3}-a_{\min } \\
& \frac{1}{5} \Delta_{3}+a_{\min }<|\Delta d|<\frac{1}{5} \Delta_{3}+a_{\max } \\
&-\frac{\Delta}{2}+a_{\min }<|\Delta s|<-\frac{\Delta}{2}+a_{\max }
\end{aligned}
$$

and

$$
\frac{3}{5} \Delta_{3}+\frac{\Delta}{2}-3 a_{\max }<\Delta \Sigma<\frac{3}{5} \Delta_{3}+\frac{\Delta}{2}-3 a_{\min }
$$

where $a_{\max }$ and $a_{\min }$ are given in (2.9a). Similarly we can obtain the constraints for other interesting observables. We note that $(2.14 \mathrm{c})$ gives a range of negative strange sea polarization

$$
-0.08<\Delta s<0
$$

\section{Numerical results and discussion}

As mentioned above, a narrowed range of $a$ can be obtained by using the NMC data. Substituting (2.2a-b) into expression $A$ in (1.6), then from (1.5b), $\bar{d}-\bar{u}$ can be written as 
function of $a$, which is plotted in Fig.2. The straight lines $\delta \equiv \bar{d}-\bar{u}=0.130,0.147$, and 0.164 are also shown. They lead to $a=0.144,0.153$, and 0.155 , thus we obtain a range of parameter $a$ determined by the NMC data

$$
0.144<a<0.155
$$

which, we call the optimum range of $a$, is much narrower than that given in $(2.9 \mathrm{~b})$. If we take $\bar{d}-\bar{u}=0.147 \pm 0.024$, the lower value of $a$ would be 0.138 , and the upper value $a=0.155$ is still the same, because in our formalism, this is physically allowed upper bound of $a$ determined by (2.2a). From the range (3.1), one obtains

$$
0.39<\epsilon<0.43
$$

The range (3.1) implies that the probability of the lowest order chiral pion fluctuation (for example $u \rightarrow d+\pi^{+}$) is of the order $15 \%$, and the range (3.2) implies that the probability $(\epsilon a)$ of the chiral kaon fluctuation (for example $u \rightarrow s+K^{+}$) is of the order $6 \%$. Under the approximation $\epsilon_{\eta} \simeq \epsilon$, the probability of the chiral $\eta$ fluctuation (for instance, $u \rightarrow u+\eta$ etc) is also of the order $6 \%$. They give restrictions to the calculation from any model wave functions. Similarly we can obtain the ranges for other observables. For instance, in the range (3.1), the predicted quark spin contents are $0.85 \leq \Delta u \leq 0.86,-0.41 \leq \Delta d \leq-0.40$, and $-0.07 \leq \Delta s \leq-0.06$. These and other model predictions are listed in Table II and Table III. For comparison, the SU(3) symmetry case [18 and SU(3) breaking results assuming the kaon suppression only [19] are also listed.

\section{Quark spin contents}

To illustrate the $a$-dependence of the quark spin contents, we plot $\Delta u,-\Delta d$ and $-\Delta s$ as functions of $a$ in Fig. 3. It can be seen that the model prediction agrees very well with most recent DIS data [30,31] in the range (3.1), see also Table III. Two remarks should be made here. First, the data of $\Delta u, \Delta d$, and $\Delta s$ listed in Table III are actually $a_{u}, a_{d}$, and $a_{s}$ - the proton matrix elements of quark axial vector currents, which are obtained from the nonsinglet axial charges $a_{3,8}$, i.e. our $\Delta_{3,8}$, and singlet axial charge $a_{0}$ defined by $a_{3}=a_{u}-a_{d}, a_{8}=a_{u}+a_{d}-2 a_{s}$, and $a_{0}=a_{u}+a_{d}+a_{s}$. The axial charges $a_{3,8}$ and $a_{0}$ are determined by using the hyperon $\beta$-decay data (2.5) and the first moment of $g_{1}\left(x, Q^{2}\right)$ (see (3.8) below) measured from polarized DIS experiments. In general, the proton matrix element of singlet axial current $a_{0}$ is sum of quark spin contribution $\Delta \Sigma$ and a gluonic term. However, the separation of terms proportional to $\Delta \Sigma$ and $\Delta G$ is arbitrary and depends upon the factorization scheme. In the Adler-Bardeen (AB) scheme, the chirality is preserved and transitions between quarks of different helicities are forbidden to any order in perturbation theory, and $a_{0}\left(Q^{2}\right)=\Delta \Sigma-n_{f} \frac{\alpha_{s}\left(Q^{2}\right)}{2 \pi} \Delta G\left(Q^{2}\right)$, where $\Delta \Sigma$ is independent of $Q^{2}, n_{f}$ is the number of active quark flavors, and $\Delta G\left(Q^{2}\right)$ is gluon polarization. For the contributions from individual quark flavors, one has $a_{q}\left(Q^{2}\right)=\Delta q-\frac{\alpha_{s}\left(Q^{2}\right)}{2 \pi} \Delta G\left(Q^{2}\right)$, ( $q=u, d, s$ in this paper), where $\Delta q$ 's are also independent of $Q^{2}$. These are the standard results of axial anomaly [6]. On the contrast, in the $\overline{\mathrm{MS}}$ (or Gauge-invariant) scheme, the chirality is not conserved and one has $a_{0}\left(Q^{2}\right)=\Delta \Sigma\left(Q^{2}\right)$, and $a_{q}\left(Q^{2}\right)=\Delta q\left(Q^{2}\right)$, where $\Delta \Sigma\left(Q^{2}\right)$ and $\Delta q\left(Q^{2}\right)$ 's are now dependent of $Q^{2}$. The anomalous gluon contribution has been absorbed into $\Delta \Sigma$ or $\Delta q$ as an effective sea contribution. We do not discuss some disputes of 
which scheme is more appropriate (for instance, see [32]), but note that $a_{0}\left(Q^{2}\right)$ and $a_{q}\left(Q^{2}\right)$ 's are scheme-independent quantities. In Table III, the chiral quark model predictions are compared to the $a_{q}\left(Q^{2}\right)$ 's rather than the scheme-dependent $\Delta q$ 's. Second, we note that in the $\mathrm{AB}$ scheme, the gluonic term is independent of $Q^{2}$ at $\mathrm{LO}$, because the $\ln Q^{2}$ growth of $\Delta G\left(Q^{2}\right)$ is compensated by the $1 / \ln \left(Q^{2}\right)$ decrease of running coupling $\alpha_{s}^{(\mathrm{LO})}\left(Q^{2}\right)$ at LO, and the singlet axial charge $a_{0}\left(Q^{2}\right)$ is independent of $Q^{2}$ at the same order (for instance see [33]). Detail analysis shows that $a_{0}$ decreases very slowly with $Q^{2}$ at NLO [34]. Hence as far as we assume that the chiral quark model results of $\Delta u, \Delta d$ and $\Delta s$ can be identified as the corresponding DIS observables $a_{u}, a_{d}$, and $a_{s}$ at the range $(0.2 \mathrm{GeV})^{2}<Q^{2}<(1.0$ $\mathrm{GeV})^{2}$, and the perturbative QCD can be used down to this low $Q^{2}$ scale, the comparison of the chiral quark model prediction with the deep inelastic polarized data is meaningful. However, although the perturbative QCD evolution approach has been successfully used down to $Q^{2} \simeq 0.23 \mathrm{GeV}^{2}$ in [35], it is not clear if the approach still holds below this $Q^{2}$. We also note that the DIS data $\Gamma_{1}^{p, n, d}\left(Q^{2}\right)$ listed in Table III do not depend on the factorization scheme.

$\bar{u} / \bar{d}, 2 \bar{s} /(\bar{u}+\bar{d}), 2 \bar{s} /(u+d)$, and $\sum \bar{q} / \sum q$

We plot $\bar{u} / \bar{d}, 2 \bar{s} /(\bar{u}+\bar{d})$, and $2 \bar{s} /(u+d)$ in Fig. 4. The CCFR data 36] are also shown. It should be noted that the CCFR data only give the ratios of the strange quark momentum to light quark or light antiquark momentum, or total antiquark momentum to total quark momentum. Defining $Q \equiv \int_{0}^{1} x q(x) d x$, the data show

$$
\begin{gathered}
\kappa \equiv \frac{2 S}{\bar{U}+\bar{D}}=0.477 \pm 0.051 \\
\eta \equiv \frac{2 S}{U+D}=0.099 \pm 0.008 \pm 0.004 \\
\frac{\sum \bar{Q}}{\sum Q}=0.245 \pm 0.005
\end{gathered}
$$

It should be noted that in our notation, $q \equiv \int_{0}^{1} q(x) d x$. If the integral $\int_{0}^{1} q(x) d x$ is finite, one has $Q=x_{0}^{(q)} q$, where the mean value theorem is used and $x_{0}^{(q)}$ is between 0 and 1 . The $x_{0}^{(q)}$ value depends on the shape of function $q(x)$. Comparing to the quark distributions, the antiquark distributions are dominate in the smaller $x$ region, hence $x_{0}^{(\bar{q})}<x_{0}^{(q)}$. For similar reason, $x_{0}^{(\bar{s})}<x_{0}^{(\bar{u}+\bar{d})}$. Hence we expect

$$
\frac{2 S}{U+D}<\frac{2 s}{u+d}, \quad \frac{S}{\bar{U}+\bar{D}}<\frac{s}{\bar{u}+\bar{d}}, \quad \frac{\sum \bar{Q}}{\sum Q}<\frac{\sum \bar{q}}{\sum q}
$$

It can be seen, from Fig.4, the model predictions of $2 \bar{s} /(\bar{u}+\bar{d})$, and $2 \bar{s} /(u+d)$, and corresponding data satisfy (3.6) very well. For the ratio $\sum \bar{q} / \sum q$, (3.6) seems not hold, but the model prediction $\sum \bar{q} / \sum q=0.238$ is very close to the data (3.5).

For the ratio $\bar{u} / \bar{d}$, as Field and Feynman suggested two decades ago [37], $\bar{d}(x)$ and $\bar{u}(x)$ have different large $x$ behavior, and $\bar{u} / \bar{d}$ may differs from $\bar{u}(x) / \bar{d}(x)$. To show this, we need to know the shape of the light antiquark sea distributions, which have not been well determined. There are several phenomenological distributions from fitting the DIS data 
in the literature (see, for instance, 38 40]). Since we do not intend to present a model calculation on the quark distributions in this paper, we simply take $x\left(\bar{u}\left(x, \mu^{2}\right)+\bar{d}\left(x, \mu^{2}\right)\right)$ and $x\left(\bar{u}\left(x, \mu^{2}\right)-\bar{d}\left(x, \mu^{2}\right)\right)$ at $\mu^{2}=0.34 \mathrm{GeV}^{2}$ from 35] to represent the chiral quark model distributions, which gives $\bar{u} / \bar{d} \simeq 0.83$ and $[\bar{u}(x) / \bar{d}(x)]_{x=0.18} \simeq 0.46$ at $Q^{2}=0.34(\mathrm{GeV})^{2}$. After the QCD evolution, the ratio $\bar{u}(x) / \bar{d}(x)$ increases and is approximately 0.53 at $Q^{2}=4$ $\mathrm{GeV}^{2}$. Hence the chiral quark model prediction $\bar{u} / \bar{d} \simeq 0.65$ is not necessarily to contradict with the NA51 result $[\bar{u}(x) / \bar{d}(x)]_{x=0.18}=0.51 \pm 0.06$. However, a more detail quantitative study on the difference between these two ratios is needed.

$f_{s}$ and $f_{3} / f_{8}$

For the ratio $f_{s}$, the model prediction agrees very well with the phenomenological values (see Table II). In Table II, $f_{s}=0.10 \pm 0.06$ is taken from [41, where $f_{s}=y /(2+y)$ and $y=1-\sigma_{0} / \sigma$, with $\sigma_{0}=35 \pm 5 \mathrm{MeV}$, and $\sigma \simeq 45 \mathrm{MeV}$. If one takes $\sigma_{0}=25 \pm 5$ $\mathrm{MeV}$, then $f_{s}=0.18 \pm 0.03$, which was used in the previous works 18 22]. The value $\left(f_{s}\right)_{\text {lattice }}=0.15 \pm 0.03$, listed in Table II, is taken from the lattice QCD calculation 42. From (3.3) and (3.4), one has $1 / \kappa+1 / \eta=(U+\bar{U}+D+\bar{D}) /(2 S)=(U+\bar{U}+D+\bar{D}) /(S+\bar{S})$, where the asumption $\bar{S}=S$ (i.e. $\int_{0}^{1} x \bar{s}(x) d x=\int_{0}^{1} x s(x) d x$ ) has been used. Considering $\sum(Q+\bar{Q})=U+\bar{U}+D+\bar{D}+S+\bar{S}$, we have

$$
\frac{2 S}{\sum(Q+\bar{Q})}=\left(1+\frac{1}{\kappa}+\frac{1}{\eta}\right)^{-1}
$$

Using the CCFR data, one obtains $0.076 \pm 0.022$ for the r.h.s. of (3.7). This number has been used in Table II. Hence our prediction $f_{s}=0.10$ satisfies $f_{s}=2 s / \sum(q+\bar{q})>2 S / \sum(Q+\bar{Q})$. For the ratio $f_{3} / f_{8}$, the model prediction is consistent with the phenomenological value $f_{3} / f_{8}=0.23 \pm 0.05$ [18].

First moments of $g_{1}^{p, n, d}$

The first moment of $g_{1}^{p}$ can be expressed in terms of the axial charges $a_{3,8}$ and $a_{0}$

$$
\Gamma_{1}^{p}\left(Q^{2}\right)=\frac{C_{\mathrm{NS}}\left(Q^{2}\right)}{12}\left(a_{3}+\frac{a_{8}}{3}\right)+\frac{C_{\mathrm{S}}\left(Q^{2}\right)}{9} a_{0}\left(Q^{2}\right)
$$

where $C_{\mathrm{NS}}\left(Q^{2}\right)$ and $C_{\mathrm{S}}\left(Q^{2}\right)$ are the QCD radiative coefficient functions [43], which depend on the strong coupling $\alpha_{s}\left(Q^{2}\right)$. Similar expression for the neutron can be obtained by changing $a_{3}$ to $-a_{3}$. For the deuteron, we use $\Gamma_{1}^{d}=\eta\left(\Gamma_{1}^{p}+\Gamma_{1}^{n}\right)$, where $\eta=0.4565$. The results are given in Table III. For SLAC data, we use $\alpha_{s}\left(5 \mathrm{GeV}^{2}\right) \simeq 0.30$ for $\Gamma_{1}^{n}$ [4], and $\alpha_{s}\left(3 \mathrm{GeV}^{2}\right) \simeq 0.35$ for $\Gamma_{1}^{p, d}$ [3]. For SMC data at $Q^{2}=10 \mathrm{GeV}^{2}$, we use $\alpha_{s}\left(10 \mathrm{GeV}^{2}\right) \simeq 0.25$. It can be seen from Table III that the chiral quark model prediction agrees very well with the existing data.

Having shown a remarkable success of the model, some comments are in order.

(1) Using the nonsinglet axial charges, which can be determined by accurate low energy hyperon $\beta$-decay data, the symmetry breaking chiral quark model results are reformulated and simplified. In this new description, only one parameter remains. In principle, this parameter can be uniquely determined by the accurate data from any spin-flavor observable, except for $\Delta_{3}$ and $\Delta_{8}$. In this paper, we choose $\bar{d}-\bar{u}$ to determine $a$, and find that the 
model with both $\mathrm{SU}(3)$ and $\mathrm{U}(1)$ breakings provides a very good description to almost all existing spin-flavor observables of the nucleon.

(2) The breaking effect arising from the suppression of kaonic fluctuation is more important than that from $\eta$ suppression. In the limiting case given in [19, $\epsilon_{\eta} \rightarrow 1$, (i.e. no $\eta$ suppression, but with $\mathrm{U}(1)$-breaking, $\zeta \neq 1$ ), the overall fit is good and better than the SU(3) symmetry case (see Table II and Table III).

(3) The optimum range of $0.144<a<0.155$ gives a restriction to the range of cutoff parameter, $2.30 \mathrm{GeV}<\Lambda<2.58 \mathrm{GeV}$ in (2.11). However, the range of $\Lambda$ may varies for different models. For instance, the range of $\Lambda$ used in [14 is $2.247-5.5 \mathrm{GeV}$, which gives $\epsilon \simeq 0.35-0.57$. This result is quite similar to (3.2).

(4) From the results given in Tables II and III, the optimum fit is around $a=0.153$, which leads to $\zeta^{\prime}=-0.233$ and $\epsilon=0.427$. It implies that the kaon and $\eta$ fluctuations are strongly suppressed, and the $\eta^{\prime}$ suppression, or effective U(1)-breaking, is even stronger. The relative probabilities of pion, kaon $(\eta)$, and $\eta^{\prime}$ fluctuations are

$$
\pi: K(\sim \eta): \eta^{\prime} \simeq 1: 0.43: 0.05
$$

Note that the probability of chiral $\eta^{\prime}$ fluctuation is $\zeta^{\prime 2} a \simeq 0.008$. In the extreme case, $a \rightarrow a_{\text {max }}$, which leads to $\zeta^{\prime} \rightarrow 0$ (see (2.2a) and (2.9)). For $a=0.155$, one has $\zeta^{\prime} \simeq-0.09$. Even in this case, $\zeta^{\prime 2} a \simeq 0.001<<1$, the agreement is almost the same as in $a=0.153$ case (see Tables II and III). Hence the $\eta^{\prime}$ plays a minor role (but it means a strong U(1)breaking !), and almost decouples with the quarks in describing the spin-flavor structure of the nucleon. This is presumably caused by a very heavy mass of $\eta^{\prime}$. It implies that a better fit to the existing data requires very small $\eta^{\prime}$ contribution. This result is significantly different from that obtained in the original $\mathrm{U}(1)$-breaking but $\mathrm{SU}(3)$-symmetry description, where $|\zeta| \simeq 1.2$ leads to an unreasonable large contribution from $\eta^{\prime}$ fluctuation, $\zeta^{2} a \simeq 0.14-0.15$ (for $a \simeq 0.10$ ), which is even larger than the pionic contribution $(\sim a)$ !

(5) In obtaining the simple relations $(2.7 \mathrm{a}-\mathrm{c})$, we have used the nonsinglet axial charges $\Delta_{3}$ and $\Delta_{8}$, and rewrite all observables into functions of $a$. Since the chiral quark model is supposed to be a good approximation only at the low scale $(0.2-0.3 \mathrm{GeV})^{2}<Q^{2}<(1 \mathrm{GeV})^{2}$, it is more reasonable to use $\Delta_{3}$ and $\Delta_{8}$, which are related to the low energy hyperon $\beta$-decay data, to reform the formalism. Our result shows that this is a more effective approach to obtain an optimum fit to data. This approach, however, cannot be applied to the SU(3) symmetry scheme due to inconsistency. The data $\Delta_{3}$ and $\Delta_{8}$ given in (2.5) do not satisfy $\Delta_{3} / \Delta_{8}=5 / 3$. Hence, in the $\mathrm{SU}(3)$ symmetry case we can only choose one of $\Delta_{3}$ and $\Delta_{8}$ (we choose $\Delta_{3}$ ) and another data (we choose $\Delta s=-0.10$ [44]) as inputs. The $\mathrm{SU}(3)$ relation $\Delta_{8}=3 \Delta_{3} / 5$ leads to $\Delta_{8}=0.754$ as shown in Table III.

(6) Finally, we note that if one uses $\Delta_{3}=1.2601 \pm 0.0025$ [28] in (2.5), then (2.6) becomes $\Delta=0.177 \pm 0.025$, which does not change our main results (2.7a-d), (2.8a-e), (2.9a), (2.12), $(2.13 \mathrm{a}-\mathrm{b})$ and $(2.14 \mathrm{a}-\mathrm{d})$, and has only minor impact on the numerical results.

\section{Summary}

Using nonsinglet axial charges $\Delta_{3}$ and $\Delta_{8}$, the chiral quark model results on quark spin-flavor contents are reformulated. Using the hyperon $\beta$-decay data, the upper and lower bounds of the spin-flavor observables are given. Special attention is paid to the comparison of the prediction with data in the quark flavor sector. We found that the model predictions 
are in good agreement with the existing data in the range $a=0.144-0.155$, which gives a constraint to the cutoff in the chiral field theory. Our result shows that the $\mathrm{SU}(3)$ symmetry breaking effects arising from the kaon suppression is important, the $\eta$ plays a 'fine turning' role, and the $\eta^{\prime}$ can even be removed from the description.

\section{Acknowledgements}

This work was supported in part by the U.S. DOE Grant No. DE-FG02-96ER-40950, the Institute of Nuclear and Particle Physics, University of Virginia, and the Commonwealth of Virginia. 
TABLE I. Quark spin contents of the proton in different models

\begin{tabular}{|c|c|c|c|}
\hline \hline \hline Quantity & This paper & Skyrme model [29] & NQM \\
\hline$\Delta u$ & $4 \Delta_{3} / 5-a$ & $4 \Delta_{3} / 7$ & $4 / 3$ \\
$\Delta d$ & $-\Delta_{3} / 5-a$ & $-3 \Delta_{3} / 7$ & $-1 / 3$ \\
$\Delta s$ & $\Delta / 2-a$ & $-\Delta_{3} / 7$ & 0 \\
$\Delta \Sigma$ & $3 \Delta_{3} / 5+\Delta / 2-3 a$ & 0 & 1 \\
\hline \hline
\end{tabular}

TABLE II. Quark flavor observables (the values with * are inputs)

\begin{tabular}{|c|c|c|c|c|c|c|}
\hline Quantity & Data & $\begin{array}{c}a=0.153 \\
\left(\mathrm{~K}, \eta, \eta^{\prime}\right)\end{array}$ & $\begin{array}{c}a=0.144 \\
\left(\mathrm{~K}, \eta, \eta^{\prime}\right)\end{array}$ & $\begin{array}{c}a=0.155 \\
\left(\mathrm{~K}, \eta, \eta^{\prime}\right)\end{array}$ & \begin{tabular}{|l|}
$a=0.136$ \\
$(\mathrm{~K}$ only $)$ \\
{$[19$}
\end{tabular} & $\begin{array}{c}a=0.10 \\
\zeta=-0.429 \\
\mathrm{SU}(3)[18\end{array}$ \\
\hline $\bar{d}-\bar{u}$ & $0.147 \pm 0.024$ & $0.147^{*}$ & $0.164^{*}$ & $0.130^{*}$ & $0.147^{*}$ & 0.095 \\
\hline $\bar{u} / \bar{d}$ & {$\left[\frac{\bar{u}(x)}{d(x)}\right]_{x=0.18}=0.51 \pm 0.06$} & 0.65 & 0.60 & 0.69 & 0.61 & 0.65 \\
\hline $2 \bar{s} /(\bar{u}+\bar{d})$ & $\frac{<2 x \bar{s}(x)>}{<x(\bar{u}(x)+d(x))>}=0.477 \pm 0.051$ & 0.69 & 0.71 & 0.65 & 0.62 & 1.64 \\
\hline $2 \bar{s} /(u+d)$ & $\frac{<2 x \bar{s}(x)>}{<x(u(x)+d(x))>}=0.099 \pm 0.009$ & 0.128 & 0.124 & 0.127 & 0.106 & 0.213 \\
\hline$\sum \bar{q} / \sum q$ & $\frac{\sum<x \bar{q}(x)>}{\sum<x q(x)>}=0.245 \pm 0.005$ & 0.235 & 0.228 & 0.238 & 0.221 & 0.214 \\
\hline$f_{s}$ & 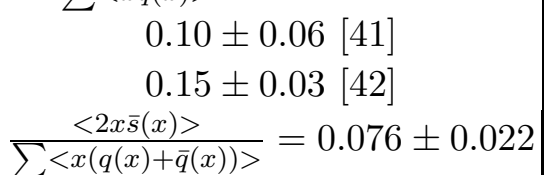 & 0.10 & 0.10 & 0.09 & 0.08 & 0.16 \\
\hline$f_{3} / f_{8}$ & $0.23 \pm 0.0518$ & 0.21 & 0.20 & 0.21 & 0.20 & $1 / 3$ \\
\hline
\end{tabular}


TABLE III. Quark spin observables (the values with * are inputs)

\begin{tabular}{|c|c|c|c|c|c|c|}
\hline Quantity & Data & $\begin{array}{c}a=0.153 \\
\left(\mathrm{~K}, \eta, \eta^{\prime}\right)\end{array}$ & $\begin{array}{c}a=0.144 \\
\left(\mathrm{~K}, \eta, \eta^{\prime}\right)\end{array}$ & $\begin{array}{c}a=0.155 \\
\left(\mathrm{~K}, \eta, \eta^{\prime}\right)\end{array}$ & $\begin{array}{c}a=0.136 \\
\text { (K only) } \\
19\end{array}$ & $\begin{array}{c}a=0.10 \\
\zeta=-0.429 \\
\mathrm{SU}(3)[18\end{array}$ \\
\hline$\Delta u$ & $\begin{array}{l}0.85 \pm 0.04 \\
0.85 \pm 0.03\end{array}$ & 0.85 & 0.86 & 0.85 & 0.87 & 0.91 \\
\hline$\Delta d$ & $\begin{array}{l}-0.41 \pm 0.04 \\
-0.41 \pm 0.03\end{array}$ & -0.40 & -0.40 & -0.41 & -0.39 & -0.35 \\
\hline$\Delta s$ & $\begin{array}{l}-0.07 \pm 0.0433 \\
-0.08 \pm 0.03\end{array}$ & -0.07 & -0.06 & -0.07 & -0.05 & $-0.10^{*}$ \\
\hline$\Delta \bar{u}, \Delta \bar{d}$ & $-0.02 \pm 0.11$ & 0 & 0 & 0 & 0 & 0 \\
\hline$\overline{\Gamma_{1}^{p}}$ & $\begin{array}{c}0.127 \pm 0.012[3] \\
0.136 \pm 0.016\end{array}$ & $\begin{array}{l}0.133 \\
0.145\end{array}$ & $\begin{array}{l}0.134 \\
0.146\end{array}$ & $\begin{array}{l}0.132 \\
0.144\end{array}$ & $\begin{array}{l}0.138 \\
0.150\end{array}$ & $\begin{array}{l}0.145 \\
0.158\end{array}$ \\
\hline$\Gamma_{1}^{n}$ & $\begin{array}{l}-0.036 \pm 0.007 \text { [4] } \\
-0.046 \pm 0.021\end{array}$ & $\begin{array}{l}-0.037 \\
-0.040\end{array}$ & $\begin{array}{l}-0.036 \\
-0.039\end{array}$ & $\begin{array}{l}-0.038 \\
-0.041\end{array}$ & $\begin{array}{l}-0.032 \\
-0.035\end{array}$ & $\begin{array}{l}-0.025 \\
-0.027\end{array}$ \\
\hline$\Gamma_{1}^{d}$ & $\begin{array}{c}0.042 \pm 0.005[3] \\
0.041 \pm 0.007\end{array}$ & $\begin{array}{l}0.045 \\
0.048\end{array}$ & $\begin{array}{l}0.046 \\
0.049\end{array}$ & $\begin{array}{l}0.044 \\
0.047\end{array}$ & $\begin{array}{l}0.049 \\
0.052\end{array}$ & $\begin{array}{l}0.056 \\
0.059\end{array}$ \\
\hline$\overline{\Delta_{3}}$ & $1.2573 \pm 0.0028$ [28] & $1.2573^{*}$ & $1.2573^{*}$ & $1.2573^{*}$ & $1.2573^{*}$ & $1.2573^{*}$ \\
\hline$\Delta_{8}$ & $0.579 \pm 0.025$ & $0.579^{*}$ & $0.579^{*}$ & $0.579^{*}$ & $0.579^{*}$ & 0.754 \\
\hline
\end{tabular}

\section{REFERENCES}

[1] J. Ashman et al. Phys.Lett. B206, 346 (1988); Nucl. Phys. B328, 1 (1989).

[2] P. L. Anthony et al. Phys.Rev.Lett. 71, 959 (1993).

[3] K. Abe et al. Phys.Rev.Lett. 74, 346 (1995); 75, 25 (1995).

[4] K. Abe et al. Phys.Rev.Lett. 79, 26 (1997); hep-ex/9705012.

[5] B. Adeva et al. Phys.Lett. B302, 533 (1993);

D. Adams et al., Phys.Lett. B329, 399 (1994); (E) B339, 332 (1994); B357, 248 (1995).

[6] G. Altarelli and G. G. Ross, Phys.Lett. B212, 391 (1988);

R. D. Carlitz, J. C. Collins, and A. H. Mueller, Phys.Lett. B214, 229 (1988);

A. V. Efremov and O. V. Teryaev, Dubna Report No. E2-88-287.

[7] K. Gottfried, Phys. Rev. lett. 18, 1174 (1967).

[8] New Muon Collaboration, P. Amaudruz et al., Phys. Rev. Lett. 66, 2712 (1991);

M. Arneodo et al., Phys. Rev. D50, R1, (1994).

[9] NA51 Collaboration, A. Baldit et al., Phys. Lett. B332, 244 (1994).

[10] J. D. Sullivan, Phys. Rev. D5, 1732 (1972).

[11] E. M. Henley and G. A. Miller, Phys. lett. 251, 453 (1990);

S. Kumano and J. T. Londergan, Phys. Rev. D44, 717 (1991);

A. Signal, A. W. schreiber, and A. W. Thomas, Mod. Phys. Lett. A6, 271 (1991);

W.-Y. P. Hwang, J. Septh, and G. E. Brown, Z. Phys. A339, 383 (1991). 
[12] H. Holtmann, A. Szczurek, and J. Septh, Nucl. Phys. A569, 631 (1996) ;

F. M. Steffens and A. W. Thomas, Phys. Rev. C55, 900 (1997)

[13] S. Brodsky and B.-Q. Ma, Phys. Lett. B381, 317 (1996).

[14] A. Szczurek, A. J. Buchmann, and A. Faessler, J. Phys. G22 1471 (1996)

[15] S. Kumano, hep-ph/9702367.

[16] A. Manohar and H. Georgi, Nucl. Phys. B234, 189 (1984).

[17] E. J. Eichten, I. Hinchliffe and C. Quigg, Phys. Rev. D45 2269 (1992).

[18] T. P. Cheng and L.-F. Li, Phys. Rev. Lett. 742872 (1995).

[19] X. Song, Preprint, INPP-UVA-96-03, March (1996),

X. Song, J. S. McCarthy, and H. J. Weber, Phys. Rev. D55 2624 (1997).

[20] X. Song, Preprint, INPP-UVA-96-05, October (1996).

[21] H. J. Weber, X. Song and M. Kirchbach, Mod. Phys. Lett. A12, 729 (1997).

[22] T. P. Cheng and L.-F. Li, hep-ph/9701248.

[23] B. Adeva et al. Phys.Lett. B369, 93 (1996).

[24] T. P. Cheng and L.-F. Li, Phys. Lett. B366 365 (1996); (E) B381, 487 (1996).

[25] F. Close and R. G. Roberts, Phys. Lett. B316 165 (1993).

[26] X. Song, P. K. Kabir and J. S. McCarthy, Phys. Rev. D54 2108 (1996).

[27] P. G. Ratcliffe, Phys. Lett. B365 383 (1996).

[28] Particle Data Group, R. M. Barnett et al., Phys. Rev. D54 1 (1996).

[29] S. Brodsky, J. Ellis, and M. Karliner, Phys. Lett. B206 309 (1988).

[30] D. Adams et al., Phys. Rev. D56, 5330 (1997); hep-ex/9702005.

[31] J. Ellis, and M. Karliner, Phys. Lett. B341 397 (1995).

[32] H. Y. Cheng, Int. J. Mod. Phys. A11, 5109 (1996).

[33] X. Song and J. Du, Phys. Rev. D40, 2177 (1989).

[34] J. Kodaria, et. al, Phys. Rev. D20, 627 (1979); Nucl. Phys. B159 99 (1979);

J. Kodaria, Nucl. Phys. B165 129 (1979).

[35] M. Gluck, E. Reya, and A. Vogt, Z. Phys. C47, 433 (1995).

[36] A. O. Bazarko, et. al, Z. Phys. C65, 189 (1995).

[37] R. D. Field and R. P. Feynman, Phys. Rev. D15, 2590 (1977).

[38] A. D. Martin, W. J. Stirling, and R. G. Roberts, Phys. Rev. D50, 6734 (1995).

[39] A. D. Martin, R. G. Roberts, and W. J. Stirling, Phys. Lett. B387, 419 (1996).

[40] H. L. Lai, et. al, Phys. Rev. D55, 1280 (1997).

[41] J. Gasser, H. Leutwyler, and M. E. Saino, Phys. Lett. B253, 252 (1991).

[42] S. J. Dong, J.-F. Lagae, and K. F. Liu, Phys. Rev. D54, 5496 (1996).

[43] S. A. Larin, Phys. Lett. B334, 192 (1994).

[44] In the $\mathrm{SU}(3)$ symmetry case, we can choose $\Delta s=-0.08$ instead of $\Delta s=-0.10$ as input, then in the flavor sector, one has $\bar{d}-\bar{u}=0.109, \bar{u} / \bar{d}=0.55$, but $2 \bar{s} /(\bar{u}+\bar{d})=1.88$. The ratios $\bar{s} /(u+d), \sum \bar{q} / \sum q$ and $f_{s}$ are almost unchanged. In the spin sector, one has $\Delta u=0.93, \Delta d=-0.33, \Delta s=-0.08$ (input), $\Gamma_{1}^{p}=0.158, \Gamma_{1}^{n}=-0.019, \Gamma_{1}^{d}=0.061$. There are no changes for the SU(3) values of $f_{3} / f_{8}$ and $\Delta_{3} / \Delta_{8}$, hence $\Delta_{8}=0.754$. 


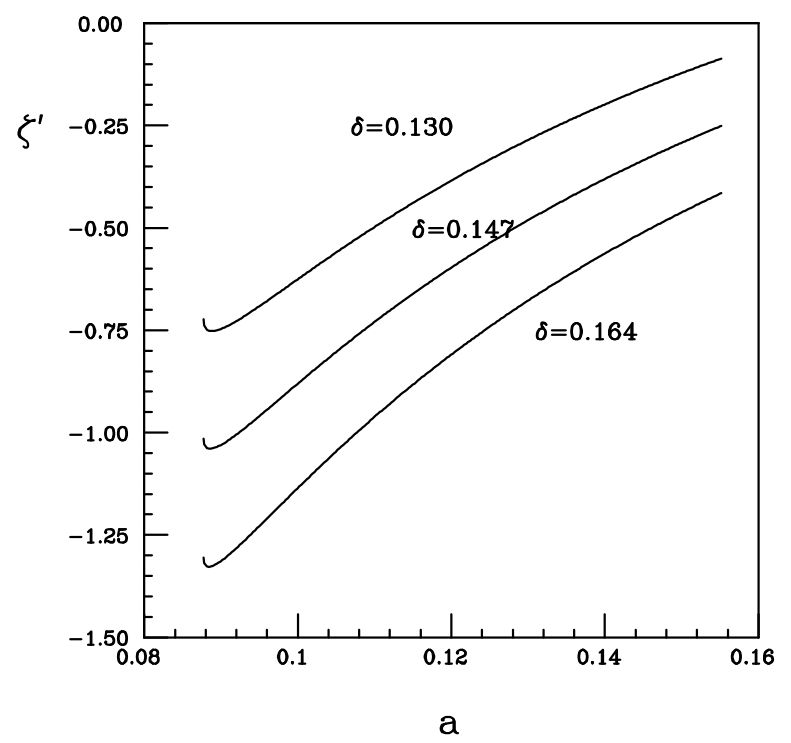

Fig. 1

FIG. 1. The U(1)-breaking parameter $\zeta^{\prime}$ as function of $a,(2.12 \mathrm{a})$, for $\delta \equiv \bar{d}-\bar{u}=0.130,0.147$, and 0.164 . 


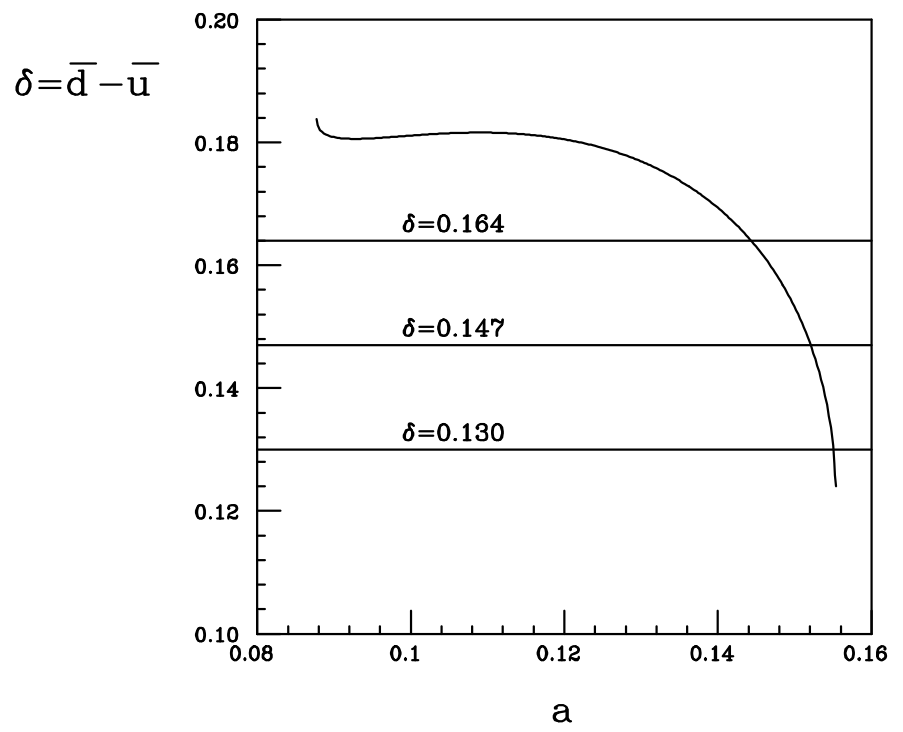

Fig. 1

FIG. 2. $\delta(a) \equiv \bar{d}-\bar{u}$ as function of $a$, from (1.5b) and (1.6). 


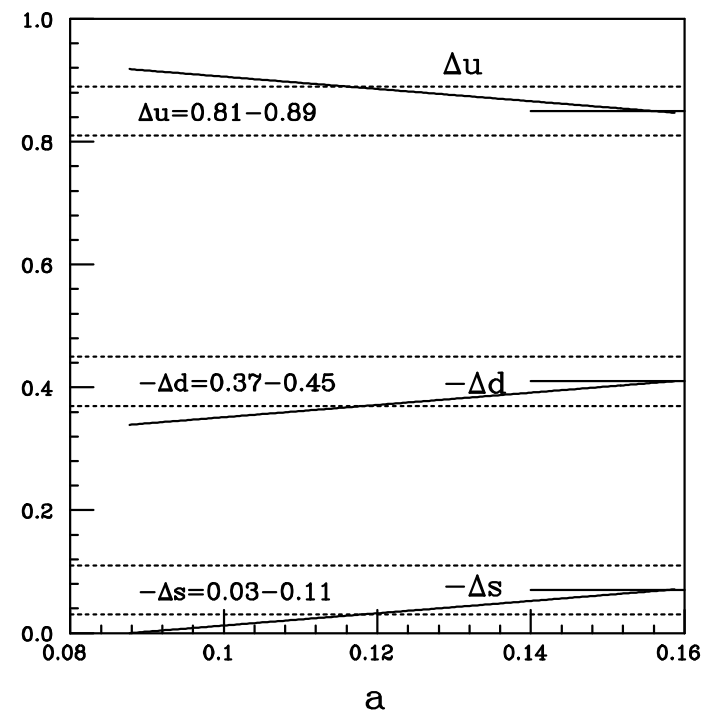

Fig.3

FIG. 3. Quark spin contents as functions of $a$ in the symmetry breaking chiral quark model. 


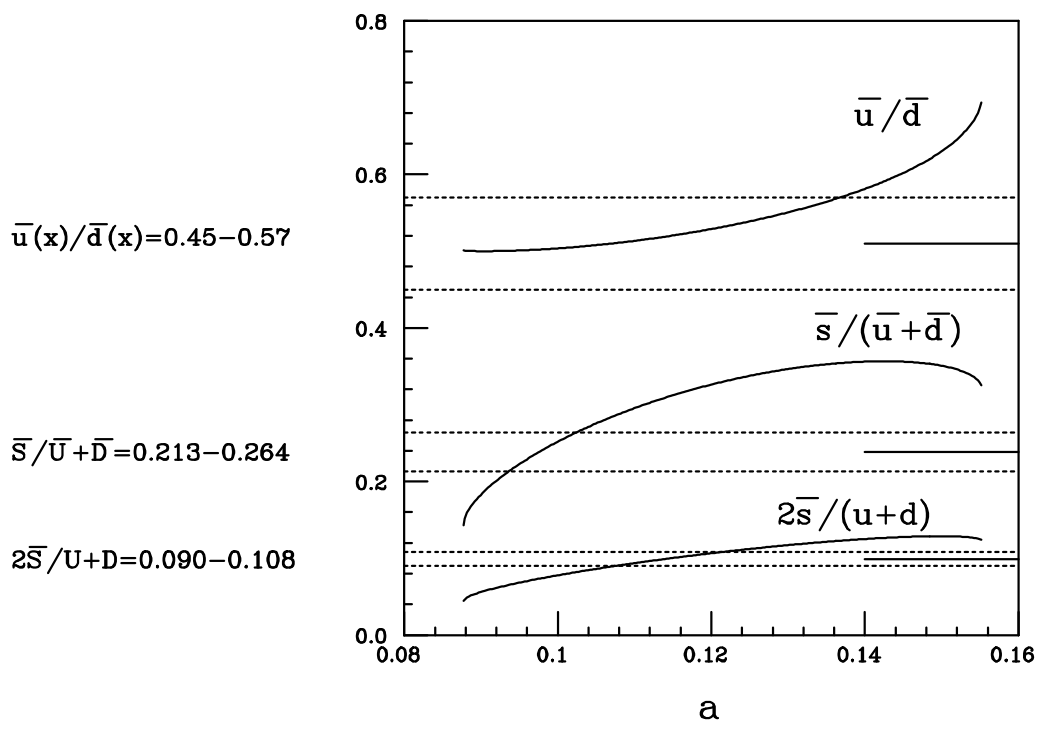

Fig.4

FIG. 4. Quark flavor observables as functions of $a$ in the symmetry breaking chiral quark model. The corresponding data and comparison with the model predictions are explained in the text. 